\title{
Developments in ultra-high energy accelerators
}

\section{A great deal of knowledge of the structure of the atomic nucleus}

has been gleaned during the past 30 years from accelerators yielding particles

of energies up to hundreds of millions of electron volts-but many mysteries

remain. "Particle physicists" are now investigating the "fundamental

particles" using protons or electrons accelerated to energies

of billions of electron volts

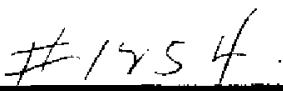

There are two main reasons for experimentation with extremely high energies. First, many ephemeral particles are produced in collisions at very high energies. Presumably a knowledge of the properties of these particles will point the way to an understanding of the behavior of nuclear matter. But some have masses greater than that of the proton and can be produced only in multibillion-volt collisions. The second reason for high energies lies in the fact that the de Broglie wavelength associated with billion-volt particles has become short compared with nuclear dimensions. With these waves it is possible to "illuminate" the interior of the proton or the neutron in a fashion that makes possible the observation of more detail. It now becomes possible to say that the density of the proton does not appear to be uniform bat may be concentrated in one or more very small cores.

For these reasons, one of the primary objectives in 1947 of the newly formed Brookhaven National Laboratory, in Upton, N.Y., was the construction of the 3-billion-volt Cosmotron. In 1952 it was completed and, for the first time in history, controlled billion-volt particles became available for experiment. Other laboratories also had set themselves the same task: in 1953 a 1-bev (billion electron volts) accelerator was completed at Birmingham in England; in 1954 a 6-bev machine began operating at the University of California in Berkeley; in 1957 the highest energy available was raised to $10 \mathrm{bev}$ in the USSR.

Until 1959, the Soviet machine provided protons of the highest energy in the world. But in 1953 construction had been started on two larger machines: at a joint European laboratory (CERN) in Geneva, Switzerland, where work was conmenced on a 28-bev machine of new design; and at Brookhaven, where a study was initiated for a 33-bev accelerator. Both machines were based on a new principle, to be dis-

Essentially full text of a conference paper presented at the AIEE Essentially full text of a conference paper presented at
Fall General Meeting, Oct. 15-20, 1961, Detroit, Mich.

J. P. Blewett is with the Brookhaven National Laboratory, Upton, N. $Y$. cussed later, discovered in 1952 at Brookhaven. In 1959, the CERN machine came into operation and last year the Brookhaven accelerator was brought to its design energy,

To complete the catalogue of billion-volt accelerators a 3-bev French machine was completed in 1958 at Saclay, outside of Paris. All of the machines mentioned thus far are synchrotrons. A linear accelerator for électrons passed 1 bev at Stanford last year, and plans are on foot for a 20 - to 40 -bev version. Six more synchrotrons are under construction: a 3-bev rapid-cycling synchrotron at Princeton University (a joint effort between Princeton and the University bf Pennsylvania); a 6-bev electron synchrotron a Harvard University (a joint HarvardMassachusetts Institute of Technology project); a 12.5-bev synchrotron at the Argonne National Laboratory; a 7-bev synchrotron at the Rutherford Laboratory in England; and 7- and 70-bev synchrotrons in the USSR.

These are all major projects. Costs range from \$1to $\$ 4$-million per bev, and hundreds of people are involved in construction and operation. Operating costs are high - the annual cost of operation and experimentation with the Brookhaven 33-bev machine will soon reach $\$ 10$ million. Fortunately, our government feels that truly fundamental research of this nature is worthy of support, and accelerator developments have been encouraged and financed by several agencies, particularly by the Atomic Energy Commission (AEC).

\section{THE SYNCHROTRON}

In the synchrotron, the particles to be accelerated arc constrained by a magnetic field to travel in a roughly circular path of constant radius. Acceleration is by $\mathrm{r}$-f fields applied at one or more points around the orbit; the frequency of the field must be the frequency of revolution of the particles or a multiple of that frequency. Since the frequency of revolu- 


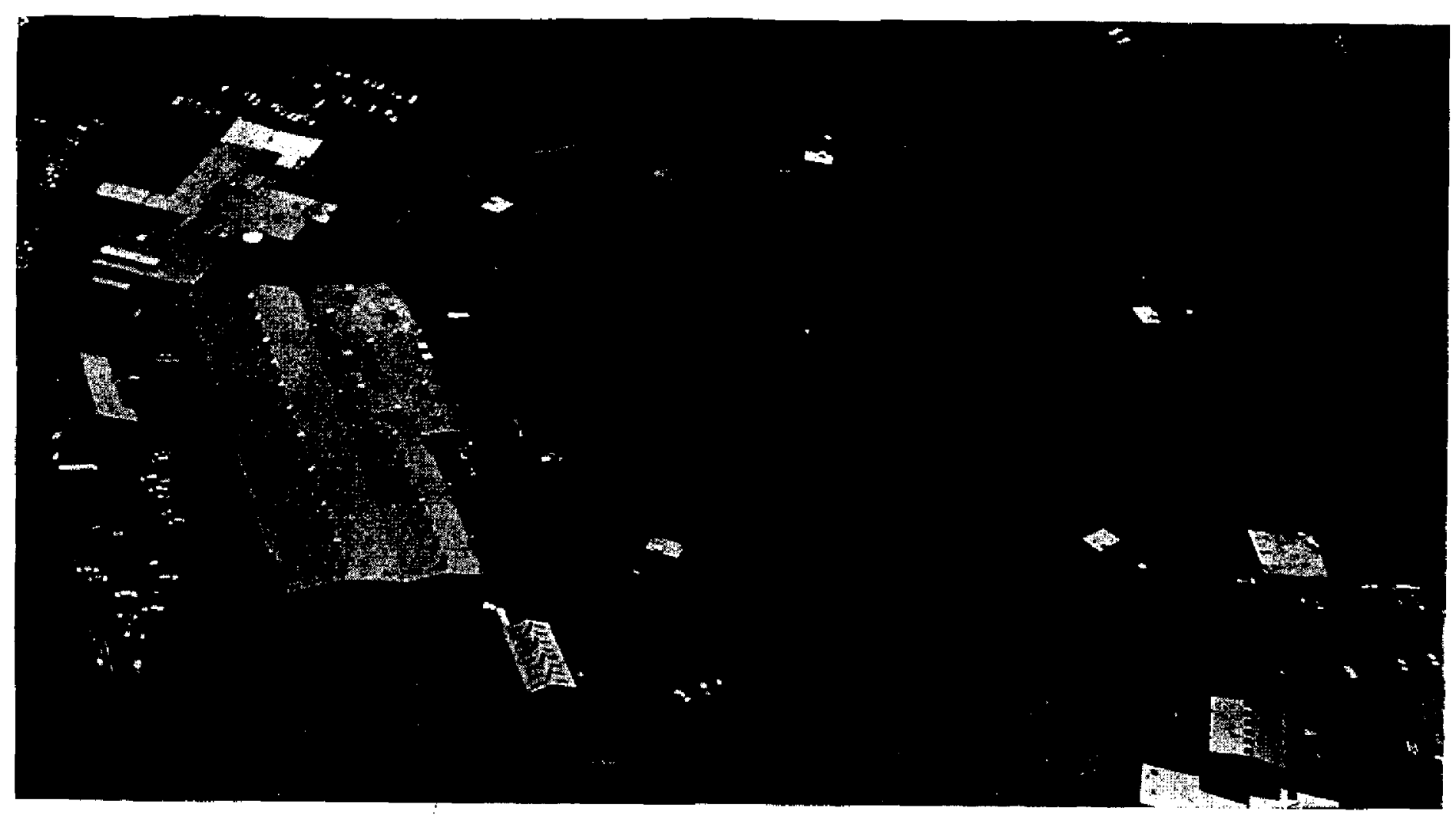

Fig. 1. Aerial view of the Brookhaven AGS

tion increases as the particle velocity increases, the frequency of the accelerating field must also change, in some machines over a factor of 10 or more, during acceleration. Obviously this has called for solution of numerous difficult problems in electronic engineering.

As the energy of the particle increases, the magnetic field is increased at such a rate as to keep the orbit radius constant. Fortunately there are several mechanisms at work to keep the particle energy in step with the applied magnetic field.

To keep the particle beam from wandering off to the vacuum chamber walls during the many thousands of revolutions required for acceleration, some focusing means are required. These are included by appropriate shaping of the magnetic field. If the field falls off with radius, but not as fast as $1 / r$, focusing will take place in both planes. This focusing is not very strong, however, and much space must be left for the orbit oscillations. In the Cosmotron, whose radius is 30 feet, the space required for the beam is about $6 \times 30$ inches. To provide good field over this volume requires a magnet about $8 \times 8$ feet in cross section and weighing a total of almost 2,000 tons. Machines of higher energies have magnets whose volume is roughly proportional to the cube of the energy; the magnet for the Russian 10-bev machine weighs 36,000 tons!

Fortunately for the future of the synchrotron a new idea was born about 10 years ago-the idea of "alternating-gradient" focusing. This method provides a solution to a fundanental difficulty that strong focusing in one plane always results in defocusing in the other. It was shown that alternate focusing and defocusing could result in a net focusing in both planes much stronger than had been achieved in the old accelerators. This discovery reduced magnet dimensions to the point that the magnet for the 30-bev accelerator weighs only about twice as much as did the magnet for the 3-bev Cosmotron. The new machine is known as the AGS, for alternating-gradient synchrotron.

\section{THE ALTERNATING-GRADIENT SYNCHROTRON}

Fig. 1 is an aerial view of the AGS ring. The circumference of the machine is almost exactly $1 / 2$ mile. For radiation shielding it is buried under 10 -feet of earth-its outline can be seen in the upper center of the picture. The large building at the left is the experimental area--this building covers almost two acres and still will be barely adequate to house the long beam paths and massive equipment required for experiments at multibillion-volt energies. The building behind the experimental area is a service and office building housing control rooms, magnet power supply, mechanical equipment. assembly areas and laboratories. The building under construction in the right foreground will house a liquid-hydrogen bubble chamber 80 inches in length. The small square building at the right-hand side of the ring covers the end of the injection tunnel.

In a pit in this building is the $750-\mathrm{kv}$ preinjector shown in Fig. 2. The protons begin their journey in a hydrogen discharge in the high-voltage terminal shown. They are accelerated to $750 \mathrm{kev}$ as they leave the terminal and enter the linear accelerator shown in 


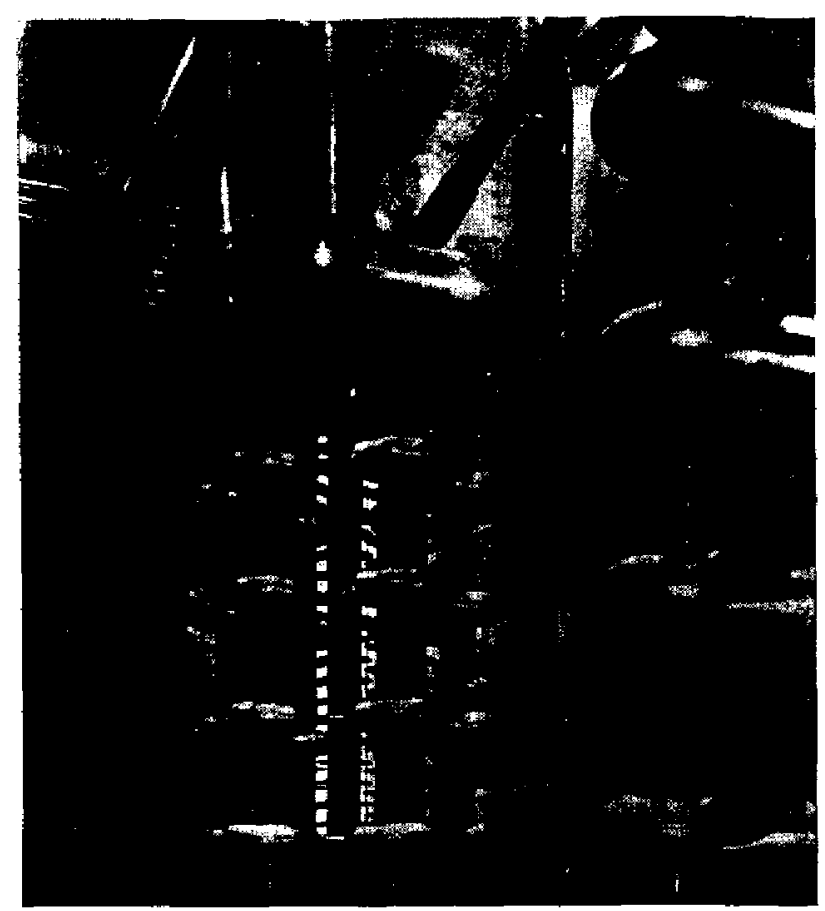

Fig. 2. View of the 750-kv Cockcroft-Walton preaccelerator for the $A G S$

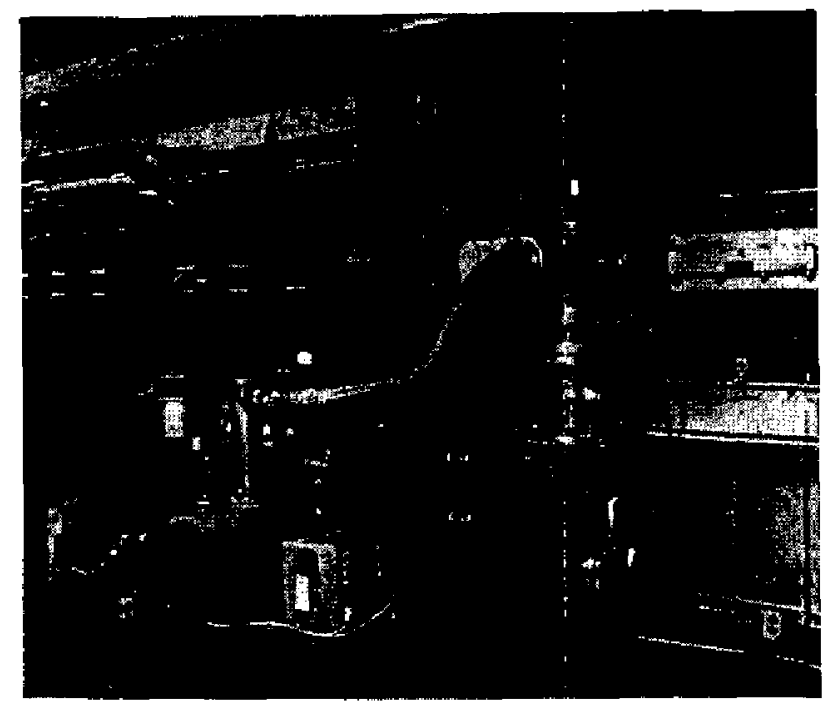

Fig. 4. Typical view in AGS magnet ring

Fig. 3. Here their energy is raised to 50 mev by $200-$ $\mathrm{mc}$ electric fields maintained between a series of drift tubes. From the linear accelerator they proceed to the magnet tunnel (Fig. 4) where they are inflected into their final orbit. Over a period of 1 second the magnetic field is raised and accelerating fields are applied at 12 points around the ring until, finally, after traveling about 180,000 .miles, their energy reaches the peak value of 33 bev. Now the beam is brought to a target and intense secondary beams of all sorts of radiation emerge in all directions. The cmergent beans pass through defining holes in massive concrete shielding to enter the various types of detecting and measuring equipment. Fig. 5 shows one of the congested experimental regions.

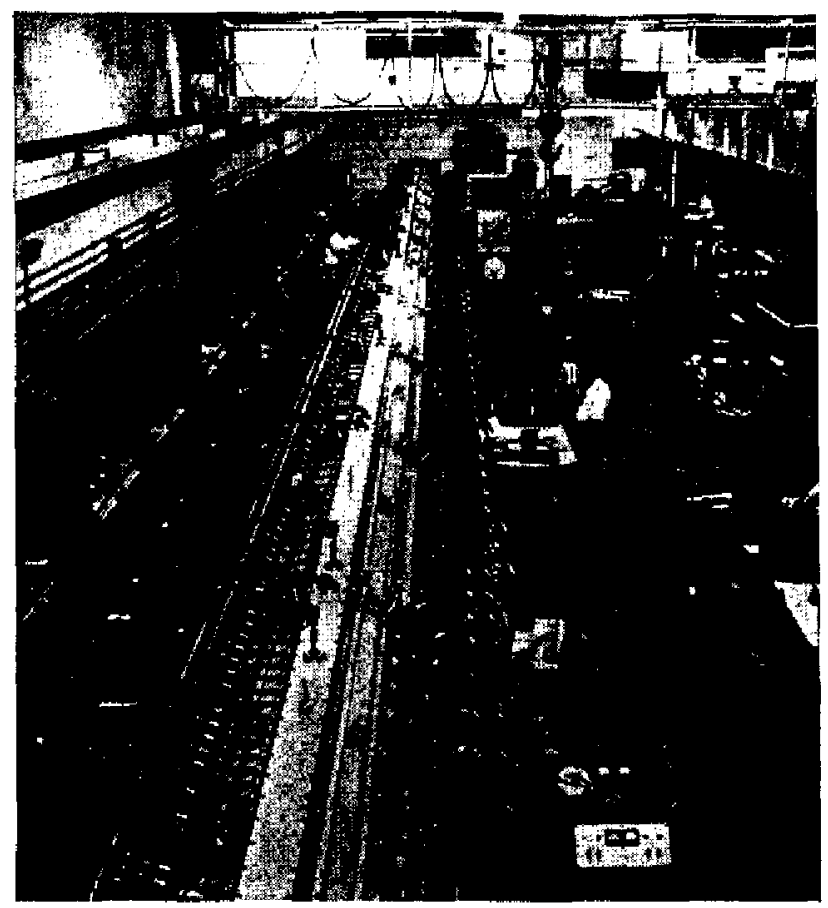

Fig. 3. View of the 50-mev linear accelerator used as injector for the $A G S$

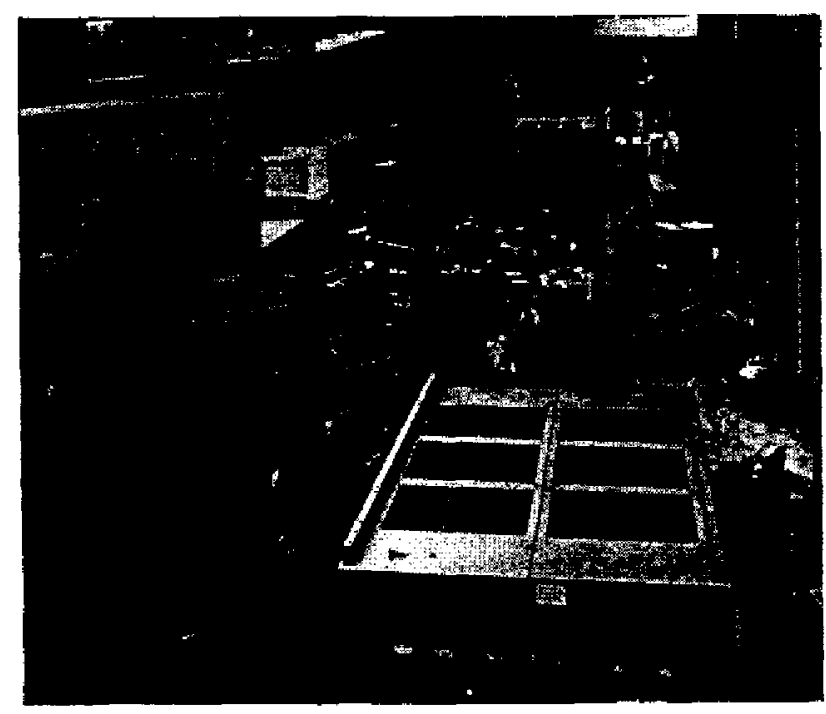

Fig. 5. AGS experimtental area

\section{THE CERN PROTON SYNCHROTRON}

Fig. 6 is an aerial view of the joint European machine in Geneva, The European Organization for Nuclear Research is known as CERN, the initials of its original name in French. The CERN group has called its machine the PS, for proton synchrotron. This machine is almost identical in principle with the AGS; it is slightly smaller and has a peak energy of $28 \mathrm{bev}$. The magnet tunnel, buried like the AGS tunnel. can be seen at the left of the picture. The large building in the left foreground is the experimental area.

\section{0-TO-300-BEV DESIGN STUDIES}

Both the Brookhaven AGS and the CERN-PS continue to yield new and important information but it 


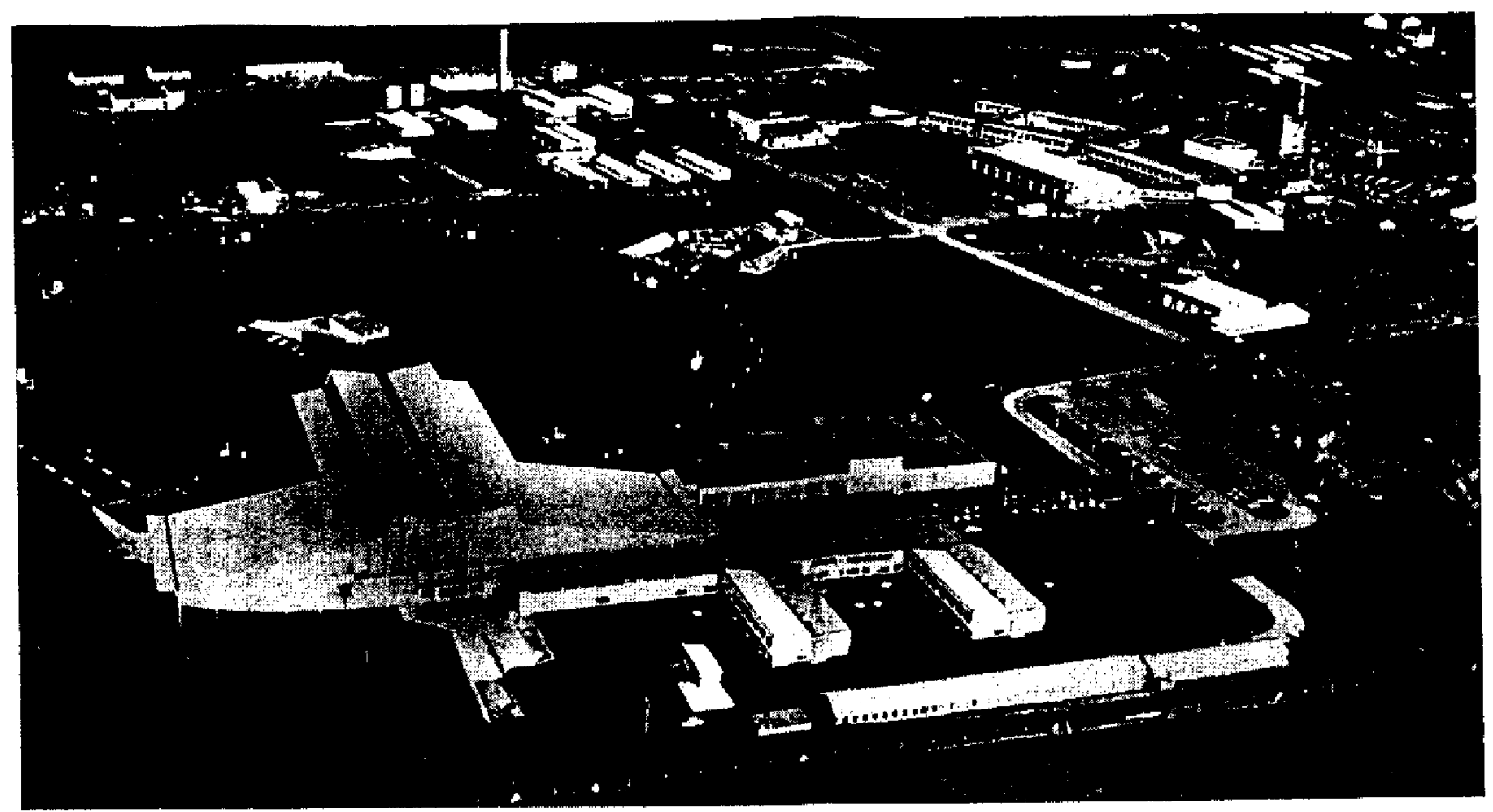

Fig. 6. Aerial view of the CERN-PS

is already evident that still higher energies will be required for solution of many of the problems exposed by the work in the present energy range. About 2 years ago, M. Sands of the California Institute of Technology (Cal Tech) proposed a 2-stage accelerator in which a machine about half the size of the AGS would be used as the injector for a 300 -bev ring. He showed that, although the circumference of the ring would be about 5 miles, the magnet cross section could be reduced below that used in the AGS and, in his first proposal, the total magnet weight for both rings added up to 6,700 tons, less than twice the weight of the AGS magnet. A study was initiated at Cal Tech and is still in progress for investigation of various design problems and details. About a year ago a similar study was started at the University of California Radiation Laboratory in Berkeley. This group, headed by Lloyd Smith, has studied the possibility of injection from a scaled-up linear accelerator and has considered machines in the range from 100 to 300 bev, A summer study was held in Berkeley during the past summer; attention was given primarily to the experimental program appropriate to such a machine and particle physicists from all parts of the United States and Europe joined in agreeing that much remains to be done and that the higher the energy attained the better.

The two California groups are in close contact and can be expected before long to amalgamate and concentrate on one machine design. Probably a 300-bev machine will cost at least $\$ 200$ million and will require a colossal effort in manpower and time. Such a machine will call for a national effort on the part of the accelerator fraternity and will become in truth a national machine.

\section{A 300- TO 1,000-BEV DESIGN STUDY}

As a result of the McCone-Emelyanov agreement that the United States and the USSR should cooperate where possible on the peaceful uses of atomic energy, there has been much discussion of the possibility of co-operation on a large accelerator. About a year ago an official meeting was held between representatives of the two countries and it was agreed that study groups should be set up in both countries to consider the design of a machine to be larger than anything presently considered in either country; this indicated a lower limit of 300 bev because of the California efforts. Responsibility for the American study was delegated by the AEC to the Brookhaven Laboratory where the writer undertook to organize this study. Our study has been in progress since last January; in a preliminary design report, parameters and design of machines up to 1,000 bev are tabulated and discussed. During last August a summer study on machine design was held at Brookhaven and a number of American and European accelerator experts met to scrutinize and amplify the Brookhaven proposals. It was concluded that, given sufficient resources of manpower and money, there is no evident upper limit to the energy that can be achieved.

A parallel study of experimental desirability and usability of 300 - to 1,000 -bev machines also is in progress at Brookhaven. It seems to be agreed that experimentation at these energies, though more cxpensive as energy increases, can still be feasible and profitable.

An international conference under Brookhaven sponsorship was held in Sept. 1961. It was expected that some of the accelerator groups in the Soviet 
Union might be present and notes on the two design studies could be compared but, for unforeseen reasons, the Soviet group were unable to attend. We hope that a meeting for comparison of opinions can be held in the near future. In the meantime, discussion is in progress in many European centers on the possibility of joining an international project for construction of a large accelerator. What will be the outcome of all of these discussions it is too early to guess.

Our design study has shown that 300- to 1,000-bev machines can yield beams of very high intensity as well as high energy. Some hair-raising problems will be associated with the radiation level and the residual radioactivity. We estimate that, even after a shutdown of a month, the radiation level around a target will be severa! thousand times tolerance; tests, repairs and component replacements will have to be done by remote control. Fortunately much experience of this kind has been gained in reactor operations. Another problem is associated with the enormous amounts of shielding necessary and with the long periods required for setting up and tearing down an experiment. To help in these problems the summer group at Brookhaven has evolved a method of "beam sidings" illustrated by Fig. 7 where the beam required for experiments can be taken along the outer track, but where, when the experiment is finished, the beam can be switched to the inside track behind 40 feet of heavy concrete shielding. While the experiment is torn down and a new one is set up-probably requiring at least two months-the beam can be used on the outer track of a siding in another experimental area.

The magnet for a 1,000-bev machine would have about half of the dimensions of the AGS magnet,

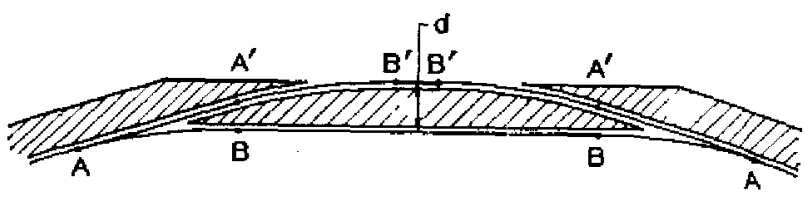

Fig. 7. Beam siding for 300-1,000 bev accelerator

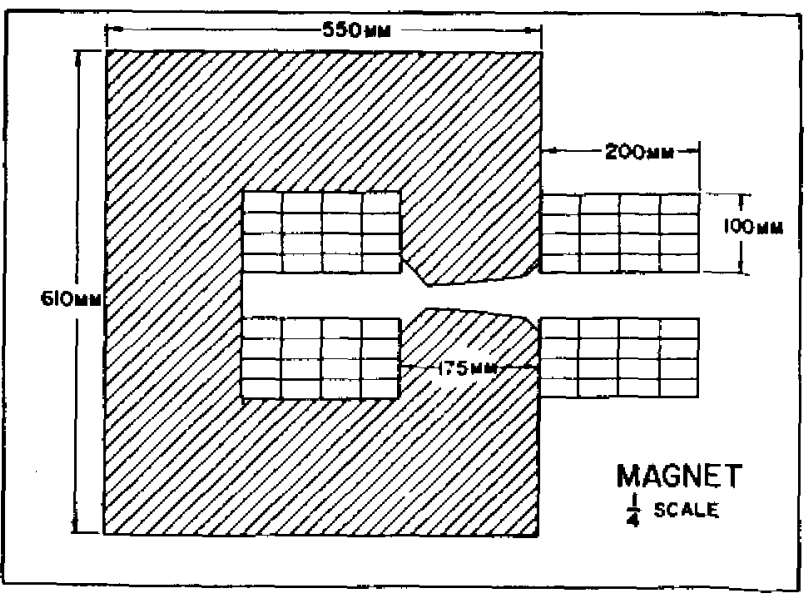

Fig. 8. Cross section of magnet for 1,000-bev accelcrator

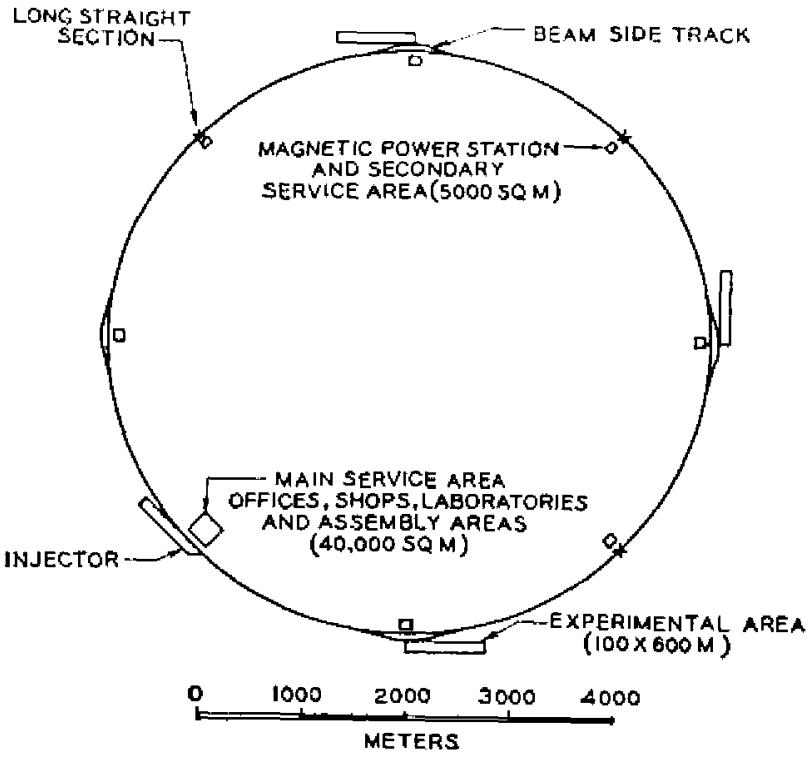

Fig. 9. Plan for 1,000-bev accelerator

which is about 3 feet square. Fig. 8 is a cross section of the proposed 1,000-bev magnet. Total magnet weight for a 1,000-bev machine would be about 30,000 tons, a considerable amount, but still less than the amount used in the Russian 10-bev accelerator.

Fig. 9 shows a possible plan for a 1,000-bev accelerator. Four beam sidings are shown-this should make possible continuous operation of the accelerator. Our present estimate is that such a machine would cost more than $\$ 700$ million. It would require a construction team of over 1,000 people. And these figures do not include any experimental gear or experimenters. The machine would be about 12 miles in circumference and each of its experimental areas would cover about 15 acres.

\section{CONCLUSION}

Whether or not the expenditure of so much money and effort in an effort to learn the fundamental properties of matter is wise or even sensible is a matter for much sober debate. It is impossible to guess what will be the eventual significance to the human race of the information obtained. In the past, extension of the bounds of human knowledge has always justified itself. Particularly in the accelerator art this has been true-the foundations of our atomic energy projects were laid on information derived from accelerators. Particle accelerators have also made many ancillary contributions. Accelerator builders have made many advances in electronics, vacuum technique, electromagnet design, instrumentation and control techniques, and further developments would certainly come from the large machines.

In the course of our design study, most of us who have been involved have concluded that a machine for at least 300 bev is desirable and worth the money. We hope that careful consideration will eventually lead our government and possibly the governments of other countries to the same conclusion. 